\title{
Clinical Outcomes of Endoscopic Submucosal Dissection for Superficial Esophageal Squamous Neoplasms
}

\author{
Jung Soo Park, Young Hoon Youn, Jae Jun Park, Jie-Hyun Kim and Hyojin Park \\ Department of Internal Medicine, Gangnam Severance Hospital, Yonsei University College of Medicine, Seoul, Korea
}

Background/Aims: Endoscopic treatment has been broadly applied to superficial esophageal neoplasms. Endoscopic submucosal dissection (ESD) allows for high rates of en bloc resection, precise histological assessment, and low rates of local recurrence. The aim of this study was to evaluate the outcomes of ESD for superficial esophageal neoplasms.

Methods: We retrospectively reviewed 36 esophageal ESDs for superficial squamous neoplasms in 32 patients between March 2009 and August 2014 at Gangnam Severance Hospital.

Results: The median patient age was 64 years, and 30 men were included. The indications were early squamous cell carcinoma in 26 lesions, adenoma with high-grade dysplasia in five lesions, and low-grade dysplasia in five lesions. The en bloc resection and R0 resection rates were $97.2 \%$ (35 of 36) and 91.7\% (33 of 36), respectively. Microperforation and post-ESD bleeding occurred in 5.6\% (2 of 36 ) and $5.6 \%$ (2 of 36), respectively. Post-ESD esophageal strictures developed in five patients (13.9\%). Five patients (15.6\%) had an additional treatment after ESD (concurrent chemoradiation therapy in three, radiation therapy in one, and surgery in one patient). There was no disease-specific mortality during the median follow-up of 31 months.

Conclusions: Favorable clinical outcomes were observed in ESD for superficial esophageal squamous neoplasms. Esophageal ESD could be a good treatment option in terms of efficacy and safety. Clin Endosc 2016;49:168-175

Key Words: Endoscopic submucosal dissection; Esophageal neoplasms; Carcinoma, squamous cell; Treatment outcome; Complications

\section{INTRODUCTION}

Endoscopic mucosal resection (EMR) has been generally applied to early gastrointestinal cancer. ${ }^{1}$ However, a critical drawback of EMR is that en bloc resection is difficult for tumors $>20 \mathrm{~mm}$, and piecemeal resection should be performed instead. $^{2}$ To overcome this and the other drawbacks not discussed here, endoscopic submucosal dissection (ESD) was developed. ${ }^{3}$ ESD allows for high rates of en bloc resection, precise histological assessment, and low rates of local recur-

Received: June 7, 2015 Revised: July 12, 2015

Accepted: July 15, 2015

Correspondence: Young Hoon Youn

Department of Internal Medicine, Gangnam Severance Hospital, Yonsei University College of Medicine, 211 Eonju-ro, Gangnam-gu, Seoul 06273, Korea Tel: +82-2-2019-3310, Fax: +82-2-3463-3882, E-mail: dryoun@yuhs.ac

(cc) This is an Open Access article distributed under the terms of the Creative Commons Attribution Non-Commercial License (http://creativecommons.org/ licenses/by-nc/3.0) which permits unrestricted non-commercial use, distribution, and reproduction in any medium, provided the original work is properly cited. rence. $^{4,5}$ Thus, ESD is widely applied for the treatment of early gastric cancer and colorectal neoplasms, and the outcomes are reported to be good. However, endoscopists need to be aware that lymph node metastasis is likely to accompany esophageal squamous cell carcinoma even in the early stage. ${ }^{6-8}$

Endoscopic treatment has been broadly applied to superficial esophageal neoplasms because of its convenience and minimal invasiveness. ${ }^{9}$ Considering that the morbidity and mortality of surgical treatment are relatively high, most patients are older in age, and the quality of life is poor after surgery, endoscopic treatment for early esophageal cancer might have considerable benefits for patients who are not expected to have lymph node metastasis. ${ }^{10}$ According to recent data, endoscopic treatment is more effective and safer than surgical resection of superficial esophageal neoplasms. ${ }^{6,11}$

However, the level of difficulty in performing esophageal ESD is high because the narrow lumen obstructs the operative view, and because of movement due to heartbeat and respiration. Additionally, there is a risk of perforation related to the 
thin muscle wall and absence of a serosal layer. Furthermore, the lumen of the esophagus is narrow, and, therefore, postESD esophageal strictures occur more frequently than strictures in the stomach. When stricture occurs, multiple sessions of endoscopic balloon dilatation (EBD) are needed. Consequently, it worsens the patients' quality of life..$^{12,13}$

As high-definition endoscopy with narrow-band imaging (NBI) was developed recently, the diagnosis of early esophageal cancer or esophageal premalignant lesions has increased. Thus, how to treat these early neoplasms has become an important issue. The existing reports on the outcomes of esophageal ESD are mainly from Japan. The aim of this report was to integrate the data from Korea where ESD has been well established. We evaluated the efficacy and safety of ESD for superficial esophageal squamous neoplasms.

\section{MATERIALS AND METHODS}

\section{Patients}

We retrospectively reviewed 36 esophageal ESDs for superficial esophageal squamous neoplasms performed in $32 \mathrm{pa}-$ tients between March 2009 and August 2014 at Gangnam Severance Hospital. Superficial esophageal squamous neoplasm was defined as an adenoma with low-grade dysplasia, high- grade dysplasia, and squamous cell carcinoma limited to the mucosal layer. Adenocarcinoma was excluded in this study to focus on squamous neoplasms that account for most of the esophageal neoplasms in the Far East regions. The Institutional Review Board of Gangnam Severance Hospital approved this study (IRB no. 2-2015-0339).

\section{Evaluation of superficial esophageal neoplasm}

The patients were evaluated by using magnifying endoscopy with NBI. We also performed chromoendoscopy with Lugol's solution. The superficial-type neoplasms were classified macroscopically into three types: 0 -I, superficial and protruding type (0-Ip, pedunculated; 0-Is, sessile); 0-II, superficial and flat type (0-IIa, slightly elevated; 0 -IIb, completely flat; 0 -IIc, slightly depressed); and 0-III, superficial and distinctly depressed type. All diagnoses were confirmed with pre-ESD histological evaluation through biopsy. Concerning the histological evaluation, in patients with superficial esophageal squamous neoplasms, we ascertained the tumor invasion depths and lymph node metastases by using endoscopic ultrasound (EUS) and computed tomography (CT). Also, we checked for distant metastasis through positron emission tomography (PET)-CT. ESD was not performed in patients suspected to have submucosal invasion, regional lymph node metastasis, or distant metastasis.
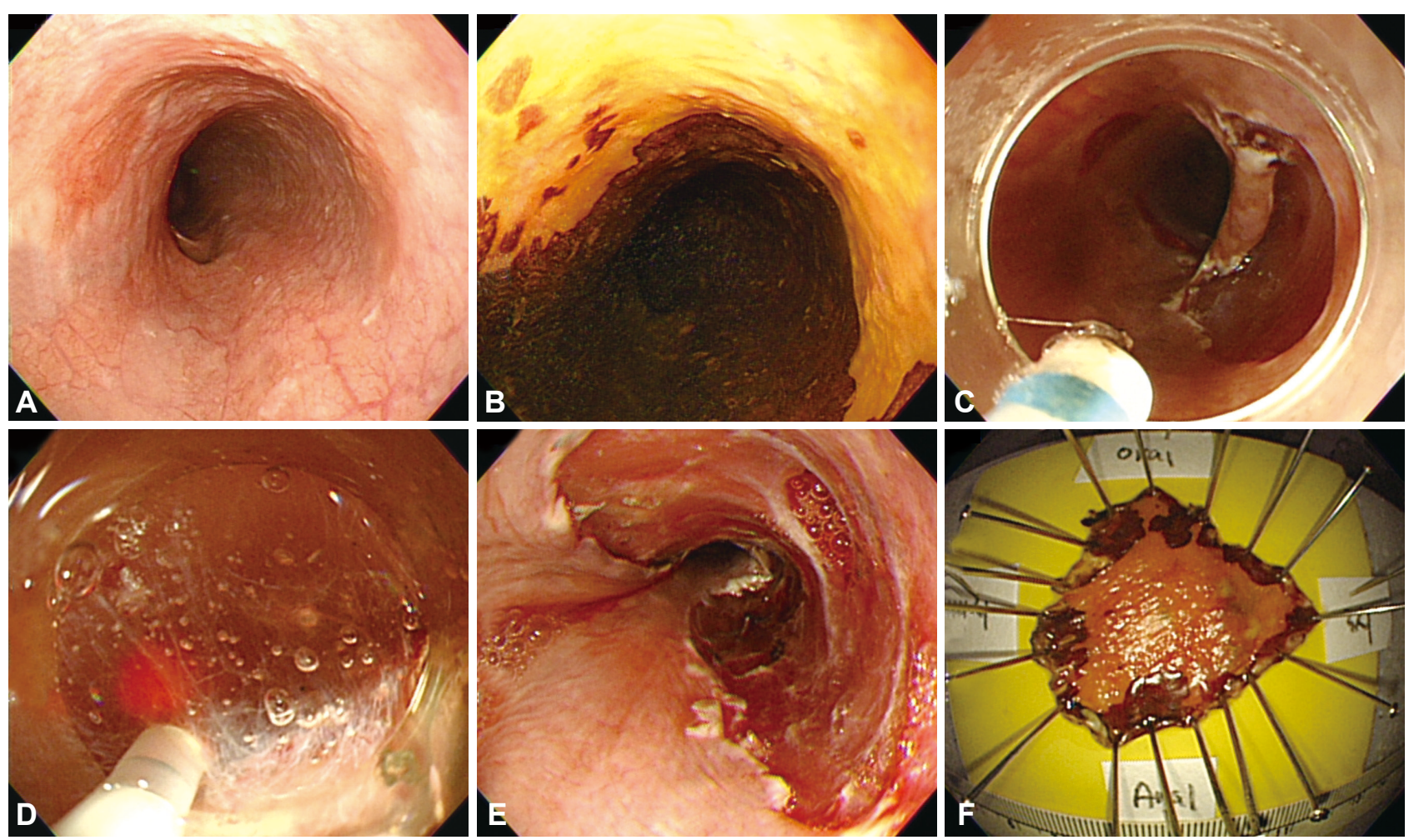

Fig. 1. Endoscopic submucosal dissection of a superficial squamous cell carcinoma. (A, B) A slightly erythematous, flat lesion that is not stained with Lugol's solution. (C, D) Submucosal dissection is made with a dual-knife after local submucosal injection. (E, F) The lesion is completely resected. 


\section{ESD procedure}

ESD was performed under moderate sedation with midazolam and propofol. A video endoscope (GIF-H260Z, GIFQ260J; Olympus, Tokyo, Japan) equipped with a 4-mm transparent cap (D-201-11804; Olympus) was used (Fig. 1).

The solution used for local submucosal injection was prepared by mixing $10 \%$ glycerol solution and $0.005 \mathrm{mg} / \mathrm{mL}$ epinephrine. In addition, hyaluronic acid (Endo-Mucoup; BMI Korea, Jeju, Korea) was used if necessary.

A dual-knife (KD-650Q; Olympus) or IT-knife 2 (KD-610L; Olympus) was used to perform the submucosal dissection with mainly the Swift coagulation mode of an electrosurgical generator (VIO 300D; Erbe Elektromedizin GmbH, Tübingen, Germany). To control bleeding, hemostatic forceps (Coagrasper, FD-410LR; Olympus) with a soft coagulation mode (60-W output) were used, and carbon dioxide $\left(\mathrm{CO}_{2}\right)$ was used for the insufflation.

Full-dose intravenous proton pump inhibitors and oral sucralfate were administered to the patient immediately after the procedure. If there was no evidence of complications such as bleeding or perforation, a liquid diet was offered on the next day, and the patient was discharged in a few days. Two skilled ESD endoscopists performed all procedures.

\section{Complications}

Microperforation was defined as radiographic evidence of free air or subcutaneous emphysema during the operation, without gross perforation defects in the esophageal wall. In the case of minor bleeding in the course of the operation, hemostasis was achieved immediately by means of thermocoagulation or hemoclipping. Massive bleeding during ESD was defined as bleeding that may necessitate the termination of the operation. Delayed postoperative bleeding was defined as a decreased blood hemoglobin level $>2 \mathrm{~g} / \mathrm{dL}$ observed 5 days after the operation, accompanied by hematemesis or melena. In addition, post-ESD esophageal stricture was defined as dysphagia after the operation that required endoscopic treatment.

\section{Histologic assessment}

The resected specimen, attached to a small plastic board and soaked in $4 \%$ formalin, was sent to a pathologist. Then, the specimen was fixed with $100 \%$ paraffin and cut into 2-mm thickness. Finally, hematoxylin and eosin staining was performed.

Histopathologic evaluation was performed to assess the tumor size, invasion depth, lymphovascular invasion, differentiation grade, and resection margin. On the basis of the invasion depth, intraepithelial cancer (m1), cancer invading the lamina propria $(\mathrm{m} 2)$, cancer invading the muscularis mucosa (m3), and cancer invading the submucosa (sm) were diagnosed.

En bloc resection was defined as removal of the lesion in one piece. Additionally, the resection margins were assessed according to the lateral and basal extensions of tumor cells as follows: R0, no cancerous cells seen microscopically; R1, cancerous cells can be seen microscopically; and Rx, not possible to establish. Also, curative resection was defined as en bloc resection with $\mathrm{R} 0$ resection for pathologically confirmed intramucosal tumor without lymphovascular invasion. The other resection was categorized as noncurative resection, and additional treatment was recommended.

\section{Follow-up}

For patients who had curative resection through ESD, follow-up upper gastrointestinal endoscopy with iodine staining was performed at 1,3 , and 6 months after the operation. Thereafter, it was performed every 6 months for 2 years, and on a yearly basis subsequently. For Lugol-voiding lesions, pathologic evaluation was performed through a biopsy. Chest and abdomen CT was performed 6 and 12 months after the operation. Moreover, PET-CT was performed at 12-month intervals.

Table 1. Clinicopathologic Features of the Patients and Tumors

\begin{tabular}{lc}
\hline Characteristic & No. (\%) \\
\hline No. of patients (no. of lesions) & $32(36)$ \\
Age, yr, median (range) & $64(42-82)$ \\
Sex & \\
Male & $30(93.8)$ \\
Female & $2(6.2)$ \\
Tumor location & \\
Upper third of the esophagus & $3(8.3)$ \\
Middle third of the esophagus & $13(36.1)$ \\
Lower third of the esophagus & $17(47.2)$ \\
Esophagogastric junction & $3(8.3)$ \\
Tumor morphology & \\
Type 0-ls & $1(2.8)$ \\
Type 0-lla & $2(5.6)$ \\
Type 0-llb & $32(88.9)$ \\
Type 0-llc & $1(2.8)$ \\
Tumor pathology & \\
Low-grade dysplasia & $5(13.9)$ \\
High-grade dysplasia & $5(13.9)$ \\
Squamous cell carcinoma & $26(72.2)$ \\
\hline
\end{tabular}




\section{RESULTS}

\section{Clinicopathological characteristics}

In total, 32 patients (median age, 64 years; range, 42 to 82 years; 30 men) were enrolled and 36 lesions were treated with ESD. Seventeen lesions (47.2\%) were located in the lower esophagus. Most of the tumors (88.9\%) were macroscopic type 0 -IIb. There were 26 squamous cell carcinoma lesions (72.2\%) (Table 1). Among these patients, two had a history of concur-

Table 2. Treatment Outcomes

\begin{tabular}{lc}
\hline Characteristic & Value \\
\hline Specimen size, mm & $28(12-64)$ \\
Tumor size, mm & $17(3-52)$ \\
Circumference of the resected specimen & \\
$<1 / 2$ & $21(60)$ \\
$<3 / 4$ & $8(22.9)$ \\
$>3 / 4$ & $6(17.1)$ \\
ESD procedure time, min & $36.5(10-240)$ \\
Tumor depth & \\
Low-grade dysplasia & $5(13.9)$ \\
High-grade dysplasia & $5(13.9)$ \\
Epithelial layer (m1) & $6(16.7)$ \\
Lamina propria (m2) & $8(22.2)$ \\
Muscularis mucosa (m3) & $7(19.4)$ \\
Submucosal layer (sm) & $5(13.9)$ \\
En bloc resection & $35 / 36(97.2)$ \\
R0 resection & $33 / 36(91.7)$ \\
Length of hospitalization, day & $4(3-13)$ \\
\hline
\end{tabular}

Values are presented as mean (range) or number (\%).

ESD, endoscopic submucosal dissection.

Table 3. Complications

\begin{tabular}{lc}
\hline Variable & Value \\
\hline Immediate complications & \\
$\quad$ Microperforation & $2 / 36(5.6)$ \\
Massive bleeding during ESD & $0 / 36(0)$ \\
Latent complications & \\
Delayed bleeding & $2 / 36(5.6)$ \\
Post-ESD esophageal stricture & $5 / 36(13.9)$ \\
$\quad$ EBD or EBD+local steroid injections & 5 \\
No. of EBD sessions & $3(2-9)$ \\
Temporary SEMS & 2 \\
\hline
\end{tabular}

Values are presented as number (\%) or mean (range). ESD, endoscopic submucosal dissection; EBD, endoscopic balloon dilatation; SEMS, self-expandable metal stent. rent chemoradiation therapy (CCRTx) for esophageal cancer and had ESD for recurrent esophageal cancer.

\section{Treatment outcomes}

The median size of the resected specimen was $28 \mathrm{~mm}$ (range, 12 to 64 ), and the median tumor size was $17 \mathrm{~mm}$ (range, 3 to 52). Six specimens extended to more than three-fourth of the circumference of the esophageal lumen. In the case of invasion depth, there were seven $\mathrm{m} 3$ lesions and five sm lesions.

En bloc resection was achieved in 35 lesions (97.2\%) and R0 resection in 33 lesions (91.7\%). For one lesion, en bloc resection could not be performed because the patient had advanced liver cirrhosis and bleeding that obstructed the operative view. Furthermore, microperforation was suspected. Finally, the procedure was ceased because of the risk of mediastinitis.

The total number of patients in whom R0 resection was not performed was three, including the above-mentioned patient. One patient had a positive basal margin ( $\mathrm{R} 1$ resection), and the other patient had extension of carcinoma in situ along the submucosal gland (Rx resection) (Table 2 ).

\section{Complications}

In the immediate complications, microperforation was observed in two lesions (5.6\%). Those two patients fully recovered with supportive medical treatment. None of the cases needed termination of ESD because of massive bleeding.

In the latent complications, delayed bleeding was observed in two lesions (5.6\%), which was successfully treated with endoscopic hemostasis. Symptomatic post-ESD esophageal strictures occurred in five lesions (13.9\%). The resected specimens of those patients extended to more than three-fourth of the circumference of the esophageal lumen. They were treated with EBD with or without local steroid injections. On average, EBD was performed three times (range, two to nine times), and a self-expandable metal stent was inserted as a temporary measure in two patients.

A gross perforation event during EBD occurred in one patient who had a post-ESD stricture. The patient was managed with temporary stenting and percutaneous endoscopic gastrostomy feeding. There was no procedure-related death (Table 3).

\section{Additional treatment}

Curative resection was performed in 25 patients (29 lesions, $80.6 \%$ ). Noncurative resection was performed in six patients; in five of these patients, the lesion invaded the sm layer, and one patient had an $\mathrm{m} 3$ tumor with lymphovascular invasion.

One patient whose lesion invaded the sm layer did not undergo additional treatment because this patient has a history of radiotherapy for breast cancer and refused additional treat- 


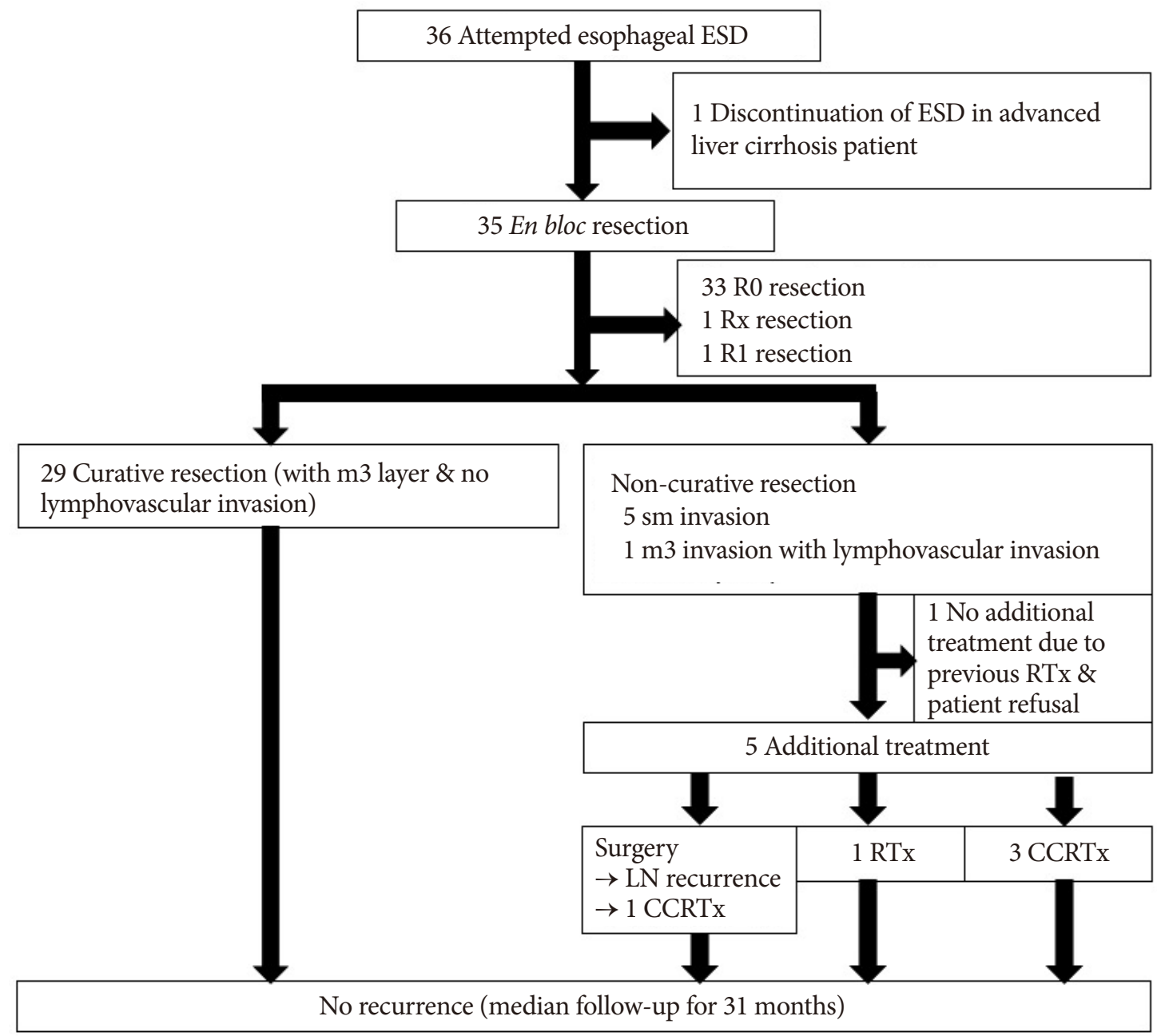

Fig. 2. Additional treatment and follow-up of 36 superficial squamous neoplasms in 32 patients who underwent endoscopic submucosal dissection (ESD). RTx, radiotherapy; LN, lymph node; CCRTx, concurrent chemoradiation therapy.

ment. The other five patients (15.6\%) had additional treatment for esophageal cancer after ESD (CCRTx in three patients, radiation therapy in one patient, and surgery in one patient). The patient who underwent esophagectomy was found to have lymph node recurrence at 24 months after the operation, and additional CCRTx was performed. Thereafter, the patient was followed without recurrence.

There was no recurrence or disease-specific mortality during a median follow-up of 31 months (Fig. 2).

\section{DISCUSSION}

ESD has been broadly applied to superficial esophageal neoplasms, and related studies have been performed mostly in Japan. Recently, there was a report of a small series in Korea. ${ }^{14}$ This study also showed that ESD is effective and safe for the treatment of superficial esophageal neoplasms.
The results showed an en bloc resection rate of $97.2 \%$ and an R0 resection rate of $91.7 \%$, which were comparable to those of precedent studies, and the rate of major complications such as perforation or stricture was also comparable to that of earlier studies. ${ }^{3,15-17}$ In addition, there was no local or distant recurrence in the median follow-up of 27 months when the patients achieved curative resection.

The most critical point in endoscopic treatment for gastrointestinal cancer is that the cancer should be completely cured with endoscopic resection. To satisfy this expectation, R0 resection needs to be performed, and at the same time, regional lymph node metastasis or distant metastasis should not exist. Unlike stomach cancer or colorectal cancer, esophageal cancer is accompanied by lymph node metastasis in the early stage, and this should be understood well before planning the treatment strategy.

According to previous studies, regional lymph node metastasis occurred in $8 \%$ to $18 \%$ of patients with $\mathrm{m} 3$ invasion and 
$17 \%$ to $53 \%$ of patients with $s m 1$ invasion. ${ }^{6-8}$ On the basis of those results, ESD for early esophageal squamous cell carcinoma limited to the $\mathrm{m} 1 / \mathrm{m} 2$ layer has been generally accepted. However, the criteria have not been established to date.

In their study of $\mathrm{m} 3$ or sm1 superficial esophageal carcinoma patients, Higuchi et al. ${ }^{18}$ reported that tumors with lymphatic invasion, a tumor size of at least $25 \mathrm{~mm}$, and a lamina muscularis mucosa invasion width of at least 2,500 $\mu \mathrm{m}$ are at a high risk for lymph node metastasis. Additionally, they pointed out that patients who do not have those tumor characteristics can be considered for endoscopic treatment even when they have $\mathrm{m} 3$ or sml invasion. ${ }^{18}$ Kim et al. ${ }^{19}$ analyzed 200 patients with $\mathrm{T} 1$ esophageal carcinoma who were treated surgically, and concluded that a tumor size $>20 \mathrm{~mm}$, endoscopically nonflat type, and lymphatic invasion were significant independent risk factors for lymph node metastasis. They suggested that considering these factors, endoscopic treatment can be performed. ${ }^{19}$ In study of Eguchi et al. ${ }^{8}$ of 464 patients with superficial squamous cell carcinoma of the esophagus who had undergone a radical esophagectomy with lymph node dissection, four of 38 patients (10.3\%) with m3 lesions without lymphatic invasion had lymph node metastasis, whereas five of 12 patients (41.7\%) with $\mathrm{m} 3$ lesions and lymphatic invasion had lymph node metastasis. The authors suggested that $\mathrm{m} 3$ lesions without lymphatic invasion can be followed after endoscopic treatment without any additional treatment. ${ }^{8}$

We considered the curative resection complete if there was no lymphovascular invasion in the resected specimen of the patients who had $\mathrm{m} 3$ invasion, and regional lymph node metastasis was not observed on EUS, CT, and PET-CT. In this study, seven patients had $\mathrm{m} 3$ invasion, and among the patients, one patient had lymphovascular invasion. The patient who had $\mathrm{m} 3$ layer and lymphovascular invasion underwent CCRTx as an additional treatment. The other six patients without lymphovascular invasion did not undergo additional treatment but were closely followed. Local or distant recurrence was not observed with the six patients in 44 months. However, there is no current standard guideline for $\mathrm{m} 3$ squamous cell carcinoma without lymphovascular invasion after en bloc resection with ESD.

Patients with sm invasion, regardless of the presence of lymphovascular invasion, are inappropriate candidates for ESD. According to previous studies, one factor that can predict sm invasion is gross tumor shape. Endo analyzed the invasion depth according to gross shape in superficial esophageal cancer. Flat-type (llb) tumors showed sm invasion in $0 \%$ (0 of 12), whereas type l, lla, llc, lll, and combined lesions showed sm invasion in 100\% (53 of 53), 66.7\% (4 of 6), 38.4\% ( 30 of 78 ), $100 \%$ ( 21 of 21 ), and $97.7 \%$ (42 of 43 ), respectively. ${ }^{20}$
In our study, the total number of patients with nonflat-type lesions was four. One (lla lesion) had a sm lesion, whereas another (ls lesion) had an $\mathrm{m} 3$ lesion with lymphatic invasion. CCRTx was performed as an additional treatment. From the case of a nonflat-type lesion, the possibility of noncurative resection is high; thus, other treatment modalities should be considered. In addition, the author of this study does not consider ESD as a primary treatment for nonflat-type lesions. The establishment of standard indications for ESD through further studies is necessary in the future.

In this study, one patient showed extension of carcinoma in situ along the submucosal gland. According to the Japanese guidelines for clinical and pathological studies on carcinoma of the esophagus, tumors with ductal involvement that extends to the submucosal gland but does not invade the submucosal stroma should not be classified as submucosal carcinoma. ${ }^{21}$ Also, Tajima et al. ${ }^{22}$ analyzed 201 surgically resected superficial squamous cell carcinomas and concluded that ductal involvement as a pathway of tumor spread to the deeper layer is of little significance in squamous cell carcinoma of the esophagus, and that mucosal carcinomas with ductal involvement that extends to the submucosa should not be classified as submucosal carcinoma. On the basis of these results, the lesion was not classified as submucosal carcinoma. However, we considered the procedure for this lesion as $\mathrm{Rx}$ resection because the submucosal glands of the lesion were not fully removed. In esophageal ESD, there might be ductal extension of tumor cells in the submucosal gland in carcinoma in situ. Thus, it is technically important that submucosal connective tissues beneath the proper esophageal glands are fully resected to evaluate the ductal extension of tumor cells. The esophageal ESD specimen from which the submucosal glands were not completely resected cannot be classified as an $\mathrm{R} 0$ resection. The patient indicated for an Rx resection in the current study did not undergo additional treatment, and local or distant recurrence was not observed during 66 months of follow-up.

In this study, five of the six patients who had noncurative resection underwent additional treatment, and one patient from that group underwent esophagectomy but showed lymph node recurrence. Then, the patient underwent CCRTx. In light of this case, surgery as an additional treatment is not the best option. Considering the surgical risk, chemotherapy or CCRTx needs to be applied depending on the patient. To date, no study has determined which modality is appropriate for patients who undergo noncurative resection as an additional treatment. Further study on this issue is necessary.

If the muscular layer is injured during SD, perforation could occur because the esophagus does not have a serosal layer. In such cases, perforation could result in mediastinitis, 
and therefore, esophageal ESD is considered a risky procedure. In this study, gross perforation did not occur; however, microperforation and accompanying subcutaneous emphysema and mediastinitis occurred in two patients (5.6\%). In previous studies, the rate of perforation during ESD was 2.6\% to $6.9 \%$, which is comparable to that of this study. ${ }^{3,16,17}$ Immediate closure through endoscopic clipping was performed in the suspected region, and those two patients recovered with supportive care such as fasting and intravenous antibiotics. Delayed bleeding was observed in the two patients (5.6\%), which was treated with endoscopic thermocoagulation and hemoclipping. The role of second-look endoscopy has not been determined in esophageal ESD, and second-look endoscopy was not performed in this study. Further study to set the role of second-look endoscopy is necessary. There was no procedure-related mortality in this study.

Esophageal ESD has lower morbidity and mortality rates than surgery; however, the candidates need to be chosen with consideration of the above complications. As previously stated, the advanced liver cirrhosis patient whose ESD had been stopped had a high tendency for bleeding as a result of thrombocytopenia and prolonged prothrombin time. Severe bleeding obstructed the operative view, and microperforation occurred as a result. Moreover, if mediastinitis had occurred, recovery from infection might have been difficult. Therefore, the procedure had to be stopped. After the procedure, preventive antibiotics were started. Mediastinitis did not occur but delayed bleeding occurred 10 days after the procedure. This candidate was inappropriate for esophageal ESD, and this case indicates the importance of careful candidate selection.

During the long-term follow-up, post-ESD stricture after esophageal ESD is a critical complication that affects patients' quality of life. According to recent studies, post-ESD stricture occurred in $5 \%$ to $17.2 \%$ of patients. ${ }^{3,23,24}$ Ono's study of 65 patients with superficial esophageal squamous cell neoplasms revealed that a circumferential extension of $>3 / 4$ and a histologic depth of up to the $\mathrm{m} 2$ layer are reliable risk factors for post-ESD stricture. ${ }^{12}$ In addition, study of Mizuta et al. ${ }^{25}$ proved that circumferential mucosal defect size is a predictive factor in post-ESD esophageal stricture, and circumferential extension $>71 \%$ of the esophageal lumen has the highest sensitivity (100\%) and specificity (97.1\%) in predicting post-ESD stricture. In this study, of the six patients in whom the lesion extended to more than three-fourth of the luminal circumference, one patient had sm invasion and underwent esophagectomy as an additive treatment. The other five patients experienced post-ESD strictures. Overall, circumferential extension is the most reliable predictive factor for post-ESD stricture, and prevention of stricture is the most critical issue among those patients.
The conventional method that has been adopted to prevent post-ESD stricture is EBD. EBD is simple and conventional; however, it generally should be performed in multiple sessions, and there is a perforation risk. ${ }^{26,27}$ Recently, preemptive intralesional steroid injections and systemic steroid administration have been adopted. In study of Hashimoto et al., ${ }^{28} 41$ consecutive patients who had semi-circumferential mucosal defects after ESD, the group who underwent endoscopic triamcinolone injections had a significantly lower incidence of stricture, the number of required EBDs was also lower, and no adverse effects or complications were associated with the triamcinolone injections. Isomoto et $\mathrm{al}^{26}$ analyzed seven patients who underwent wholly circumferential ESD for superficial esophageal squamous cell carcinoma and compared the prophylactic EBD group and the oral prednisolone administration group. The treatment in the latter group was more effective in controlling post-ESD strictures and in preventing repeated $\mathrm{EBD}$, and was not accompanied by adverse events. ${ }^{26}$ Recently, new technology with biodegradable stents or autologous oral mucosal sheets has been reported. ${ }^{29,30}$ It is important to apply proper prevention methods, considering the lesion and the characteristics of the patients. Preemptive EBD was the conventional modality at the time when postESD strictures occurred in the patients of this study. Therefore, preemptive local steroid injections or systemic steroid administration could not be performed. Preemptive EBD was applied to all patients. Currently, they are being followed without a recurrence of symptoms.

In conclusion, this study shows favorable clinical outcomes in ESD for superficial esophageal squamous neoplasms, and reveals that esophageal ESD is a relatively safe, technically feasible, and effective treatment. Additional treatment needs to be considered after assessing the tumor invasion depth, presence of R0 resection, and presence of lymphovascular invasion. Additionally, in patients who undergo widespread circumferential resection, paying attention to prevent strictures is important. Although further massive studies with longterm follow-up are needed, ESD should be considered as the useful treatment option for superficial esophageal squamous neoplasms.

\section{Conflicts of Interest}

The authors have no financial conflicts of interest.

\section{REFERENCES}

1. Sano T, Kobori O, Muto T. Lymph node metastasis from early gastric cancer: endoscopic resection of tumour. Br J Surg 1992;79:241-244.

2. Oka S, Tanaka S, Kaneko I, et al. Endoscopic submucosal dissection for residual/local recurrence of early gastric cancer after endoscopic mucosal resection. Endoscopy 2006;38:996-1000. 
3. Takahashi H, Arimura Y, Masao H, et al. Endoscopic submucosal dissection is superior to conventional endoscopic resection as a curative treatment for early squamous cell carcinoma of the esophagus (with video). Gastrointest Endosc 2010;72:255-264.

4. Isomoto $\mathrm{H}$, Shikuwa $\mathrm{S}$, Yamaguchi $\mathrm{N}$, et al. Endoscopic submucosal dissection for early gastric cancer: a large-scale feasibility study. Gut 2009;58:331-336.

5. Fujishiro M. Perspective on the practical indications of endoscopic submucosal dissection of gastrointestinal neoplasms. World J Gastroenterol 2008;14:4289-4295.

6. Kodama M, Kakegawa T. Treatment of superficial cancer of the esophagus: a summary of responses to a questionnaire on superficial cancer of the esophagus in Japan. Surgery 1998;123:432-439.

7. Araki K, Ohno S, Egashira A, Saeki H, Kawaguchi H, Sugimachi K. Pathologic features of superficial esophageal squamous cell carcinoma with lymph node and distal metastasis. Cancer 2002;94:570-575.

8. Eguchi T, Nakanishi Y, Shimoda T, et al. Histopathological criteria for additional treatment after endoscopic mucosal resection for esophageal cancer: analysis of 464 surgically resected cases. Mod Pathol 2006;19:475-480.

9. Soetikno R, Kaltenbach T, Yeh R, Gotoda T. Endoscopic mucosal resection for early cancers of the upper gastrointestinal tract. J Clin Oncol 2005;23:4490-4498.

10. Pech O, May A, Rabenstein T, Ell C. Endoscopic resection of early oesophageal cancer. Gut 2007;56:1625-1634.

11. Fujita H, Sueyoshi S, Yamana H, et al. Optimum treatment strategy for superficial esophageal cancer: endoscopic mucosal resection versus radical esophagectomy. World J Surg 2001;25:424-431.

12. Ono S, Fujishiro M, Niimi K, et al. Predictors of postoperative stricture after esophageal endoscopic submucosal dissection for superficial squamous cell neoplasms. Endoscopy 2009;41:661-665.

13. Fujishiro M, Yahagi N, Kakushima N, Kodashima S, Ichinose M, Omata M. En bloc resection of a large semicircular esophageal cancer by endoscopic submucosal dissection. Surg Laparosc Endosc Percutan Tech 2006;16:237-241.

14. Joo DC, Kim GH, Park do Y, Jhi JH, Song GA. Long-term outcome after endoscopic submucosal dissection in patients with superficial esophageal squamous cell carcinoma: a single-center study. Gut Liver 2014;8:612-618.

15. Oyama T, Tomori A, Hotta K, et al. Endoscopic submucosal dissection of early esophageal cancer. Clin Gastroenterol Hepatol 2005;3(7 Suppl 1):S67-S70.

16. Fujishiro M, Yahagi N, Kakushima N, et al. Endoscopic submucosal dissection of esophageal squamous cell neoplasms. Clin Gastroenterol Hepatol 2006;4:688-694.

17. Ishihara R, Iishi H, Uedo N, et al. Comparison of EMR and endoscopic submucosal dissection for en bloc resection of early esophageal cancers in Japan. Gastrointest Endosc 2008;68:1066-1072.

18. Higuchi K, Tanabe S, Koizumi W, et al. Expansion of the indications for endoscopic mucosal resection in patients with superficial esophageal carcinoma. Endoscopy 2007;39:36-40.

19. Kim DU, Lee JH, Min BH, et al. Risk factors of lymph node metastasis in T1 esophageal squamous cell carcinoma. J Gastroenterol Hepatol 2008;23:619-625.

20. Endo M. Endoscopic resection as local treatment of mucosal cancer of the esophagus. Endoscopy 1993;25:672-674.

21. Japanese Society for Esophageal Diseases. Guidelines for clinical and pathologic studies on carcinoma of the esophagus, ninth edition. Preface, general principles, part I. Esophagus 2004;1:61-88.

22. Tajima Y, Nakanishi Y, Tachimori Y, et al. Significance of involvement by squamous cell carcinoma of the ducts of esophageal submucosal glands. Analysis of 201 surgically resected superficial squamous cell carcinomas. Cancer 2000;89:248-254.

23. Repici A, Hassan C, Carlino A, et al. Endoscopic submucosal dissection in patients with early esophageal squamous cell carcinoma: results from a prospective Western series. Gastrointest Endosc 2010;71:715-721.

24. Higuchi K, Tanabe S, Azuma M, et al. A phase II study of endoscopic submucosal dissection for superficial esophageal neoplasms (KDOG 0901). Gastrointest Endosc 2013;78:704-710.

25. Mizuta H, Nishimori I, Kuratani Y, Higashidani Y, Kohsaki T, Onishi S. Predictive factors for esophageal stenosis after endoscopic submucosal dissection for superficial esophageal cancer. Dis Esophagus 2009;22:626631.

26. Isomoto $\mathrm{H}$, Yamaguchi N, Nakayama T, et al. Management of esophageal stricture after complete circular endoscopic submucosal dissection for superficial esophageal squamous cell carcinoma. BMC Gastroenterol 2011;11:46

27. Ezoe Y, Muto M, Horimatsu T, et al. Efficacy of preventive endoscopic balloon dilation for esophageal stricture after endoscopic resection. J Clin Gastroenterol 2011;45:222-227.

28. Hashimoto S, Kobayashi M, Takeuchi M, Sato Y, Narisawa R, Aoyagi Y. The efficacy of endoscopic triamcinolone injection for the prevention of esophageal stricture after endoscopic submucosal dissection. Gastrointest Endosc 2011;74:1389-1393.

29. Saito Y, Tanaka T, Andoh A, et al. Novel biodegradable stents for benign esophageal strictures following endoscopic submucosal dissection. Dig Dis Sci 2008;53:330-333.

30. Ohki T, Yamato M, Murakami D, et al. Treatment of oesophageal ulcerations using endoscopic transplantation of tissue-engineered autologous oral mucosal epithelial cell sheets in a canine model. Gut 2006;55:17041710 . 